Celal Bayar University Journal of Science

\title{
Comparison of Cellular Autofluorescence Patterns of Two Model Microalgae by Flow Cytometry
}

\author{
Ugur Uzuner ${ }^{1 *(i D)}$ \\ ${ }^{1}$ Department of Molecular Biology and Genetics, Faculty of Science, Karadeniz Technical University, Trabzon, \\ TURKEY \\ *uguruzuner@ktu.edu.tr \\ *Orcid: 0000-0002-5308-3730 \\ Received: 19 November 2020 \\ Accepted: 19 April 2021 \\ DOI: $10.18466 / 827615$
}

\begin{abstract}
Microalgae are widely used in biotechnological research, especially for the production of biochemical compounds, antioxidants, secondary metabolites, pigments, carbohydrates, proteins and lipids. Various analytical methods are needed throughout both experimental and downstream processing of industrial microalgae products. As one of these methods, flow cytometry is an advantageous option for detecting fluorescently labeled recombinant proteins, lipids and metabolic compounds. It is important to take into account the autofluorescent properties of specific compartments of target cells to well establish a distinct labeling protocol during such analytical processes. Because the amount of autofluorescence may interfere with the fluorescent signal detection of specifically labeled protein or lipid content, this can prevent the precise signal detection of labeled molecules. Furthermore, it can lead to an overestimation of the amount of labeled compounds in the cells. In this study, the autofluorescent properties of two freshwater model microalgae Chlamydomonas reinhardtii (CC-124) and Chlorella vulgaris (CV-898), both of which are predominantly used in industry, were examined by flow cytometry measurements. The experimental findings revealed that fluorescent channel-2 (FL2-H) stands as the most suitable channel to achieve minimal autofluorescence of both CC-124 and CV-898 microalgae strains. The obtained results highlight that one should pay attention to the autofluorescence signals in CC-124 and CV-898 cell lines during the flow cytometry-based detection of biological products when deciding on fluorophore.
\end{abstract}

Keywords: autofluorescence, cellular morphology, flow cytometry, microalgae

\section{Introduction}

Microalgae are widely preferred eukaryotic microorganisms in biotechnology as they can produce health promoting lipids, antioxidants, polysaccharides, proteins, secondary metabolites, vitamins and pigments [1]. As the evolutionary ancestors of terrestrial plants, they can be easily cultivated at laboratory conditions in the presence of natural sunlight, atmospheric $\mathrm{CO}_{2}$, varieties of different low-cost carbon sources and the infinitesimal amounts of trace elements [2]. They currently provide numerous advantages to industrial biotechnology as food and feed supplies with high protein content, feedstocks for different biofuels production processes and sustainable bioremediation agents for municipal and urban wastewaters, all of which make microalgae essential for next generations [3, 4]. As microalgae genome manipulation tools are pretty much well established, the metabolic engineering efforts on industrially promising microalgae strains

become relatively common $[5,6]$. Microalgae are of growing interest towards various mass production systems, particularly for bioenergy, lucrative metabolites, antioxidants, nutraceuticals and pharmaceuticals [7, 8]. In addition, the specific use of photosynthetic microalgae as recombinant production hosts comprises the mass production of value-added compounds, anticarcinogenic and antimicrobial drugs, monoclonal antibodies, pharmaceutical proteins, vaccines, drug additives and various compounds used in cosmetics $[9,10]$. On the other hand, it is very important to carry out comprehensive analytical, biochemical and molecular analyzes on newly discovered microalgae strains with reliable analytical methods to reveal their industrial use and commercialization potentials biotechnologically. [11]. 
During cultivation and downstream processes, the native biological characteristics of microalgae can be disclosed by employing different analytical methods, such as fluorescence and/or electron microscopy, quantitative metric measurements, multi-dimensional single-cell analysis and molecular biology-based diagnostic tools [12]. Recently, different cell sorting mechanisms combined with flow cytometry have been developed and successfully applied to microalgae cultures to eliminate various culture contaminants including bacteria [13]. Electrical microfluidics chips with advanced separation and recognition characteristics were also adapted for microalgae cultures to recognize, label and eventually eliminate culture contaminants [14]. In addition, direct electrical detection of a single bacterium in drinking water could be a highly practical technique for ensuring the contamination-free maintenance and sustainability of open pond microalgae production systems in the future. [15].

Flow cytometry is one of the high-throughput instruments to quantitatively examine the cell size, granularity, heterogeneity of microalgae cells and related culture populations using fluorescence beams [16]. Flow cytometry provides visualization and classification of cells within morphological shapes and analysis of fluorophore-tagged signals of the molecule of interest such as proteins, carbohydrates and lipids. Preparation of cells for analysis by flow cytometry is performed based on immunolabeling approaches. One of the foremost methodological key points during standard flow cytometry analysis is to initially detect the autofluorescence of cells. Autofluorescence is defined as inherent cellular fluorescence derived by the emission of natural compounds within cells, such as chlorophyll and NADH [17]. Such interference of autofluorescence with the specific label was also reported from certain types of mammalian cells [18]. However, to the best of our knowledge, no scientific report has yet been released regarding the natural autofluorescence spectra of the freshwater microalgae strains examined, suggesting the requirement of an optimization step during the flow cytometry-based experimental and analytical processes. This study thus aimed to unravel the cellular autofluorescence spectra of two model microalgae Chlamydomonas reinhardtii (CC-124) and Chlorella vulgaris Beijerinck (CV-898) cells.

\section{Materials and Methods}

\subsection{Microalgae Culturing and Flow Cytometry Analysis}

Chlamydomonas reinhardtii (CC-124: CCALA No: 928) and Chlorella vulgaris Beijerinck (CV-898: CCALA No: 898) strains were obtained from Culture Collection of Autotrophic Organisms (CCALA) at Dukelska, Czech Republic. Both strains were immediately enriched in TAP broth or agar plates. $1 \times 10^{6}$ microalgae cells were then taken and incubated within $100 \mathrm{ml}$ modified TAP (Tris-Acetate-Phosphate) medium on an orbital shaker at room temperature and $120 \mathrm{rpm}$ shaking speed for 5 days. At least 500.000 cells (counted by Countess II FL Automated Cell Counter, Thermofisher), in the presence of over $96 \%$ cell viability detected by hematocytometerbased counting, either in the phosphate-buffered saline buffer (1xPBS) or in TAP media were run by flow cytometer, C6 Accuri (from BD Biosciences). Cells were visualized by forward scatter (FSC)-side scatter (SSC) dot plot, and then analyzed by fluorescence channels; FL1-H, FL2-H, FL3-H and FL4-H. Table 1 reveals the excitation and emission values and filters for each channel. 1 XPBS and TAP medium alone were also run to understand the background fluorescence of the cell suspensions. Figure 1 represents the wavelengths of each channel. The cell morphology was assessed by FSC (Xaxes) and SSC (Y-axes) values (as mean, geometric mean and median), representing the cell size and granularity, respectively. The fluorescence signals of at least 500.000 cells were collected through the employment of a $488 \mathrm{~nm}$ laser beam and further analyzed using different filters as shown in Table 1. The autofluorescence patterns of gated cells (P1) were also revealed in the charts by fluorescence channels (FL1-H, FL2-H, FL3-H and FL4-H). Both PBS and TAP medium were also analyzed as blank controls to the cell samples. At least 10.000 events were analyzed for each triplicate experimental run.

Table 1. Excitation and emission of fluorescence channels

\begin{tabular}{|l|c|c|c|c|}
\hline & FL1-H & FL2-H & $\begin{array}{l}\text { FL3- } \\
\text { H }\end{array}$ & FL4-H \\
\hline $\begin{array}{l}\text { Excitation } \\
\text { (nm) }\end{array}$ & 488 & 488 & 650 & 470 \\
\hline $\begin{array}{l}\text { Emission } \\
\text { (nm) }\end{array}$ & $500-550$ & $550-600$ & $\begin{array}{c}650- \\
700\end{array}$ & 650 \\
\hline Filter & $\begin{array}{c}533 / 30 \\
\text { (530 BP) }\end{array}$ & $\begin{array}{c}585 / 40 \\
(585 \mathrm{BP})\end{array}$ & $\begin{array}{c}670 \\
\text { LP }\end{array}$ & $\begin{array}{c}675 / 25 \\
(675 \mathrm{BP})\end{array}$ \\
\hline
\end{tabular}

\section{Results and Discussion}

Recent advances in recombinant DNA technology and genome editing tools have increased R\&D initiatives on microalgae strains with renewable, sustainable and economic potentials towards versatile production of various value-added products, secondary metabolites, natural pigments, antioxidants, hydrocarbons, biofuels and derivatives. On the other hand, innovative approaches mostly relying on metabolic engineering and systems biology to develop microalgae strains with improved properties yield abundant recombinants that necessitate extensive screening efforts for targeted product(s). Comparative screening and quantitative analysis of natural and/or recombinantly engineered microalgae cells is of paramount importance for the final selection and full characterization of the most promising strains. In this regard, flow cytometry is a simple 


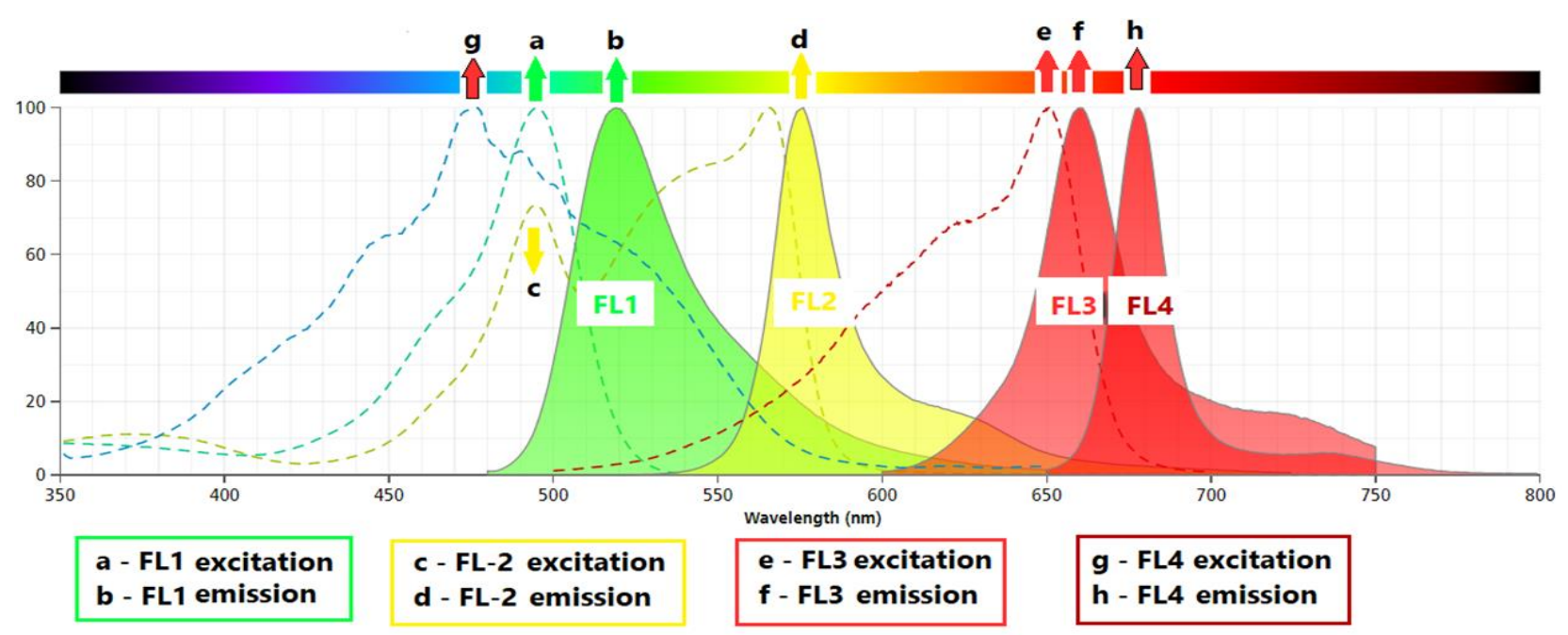

Figure 1. Representative wavelengths for emission and excitation of fluorescence channels 1, 2, 3 and 4 of BD Accuri C6 instrument using FITC, PE, APC and PerCP, respectively (modified from BD Biosciences's web site). The spectra can be modified according to dye type.

operational and analytical platform for the quantification of various microalgae cell components and related byproducts. To specifically analyze, label and even separate microalgae single cells, numerous high throughput methods with advanced analytical potentials including flow cytometry, electrical flow cytometry, electrical microfluidics chip and fluorescence-activated cell sorting have recently been reported [14, 15, 19, 20]. Among them, flow cytometry allows automatic, low-cost, highspeed and simultaneous analysis of multiple parameters of microalgae cells through qualitative and quantitative measurements of biological and physical characteristics.

Two model microalgae isolates with the adaptation to the cool temperate environments were examined to identify their cellular autofluorescence patterns by flow cytometer, C6 Accuri. The comparative run revealed that CC-124 microalgae cells were larger than CV-898 by the mean of events at FSC axes and the granularity of CC124 was more intense than $\mathrm{CV}-898$ by the mean of events at SSC axes. The median values were close to the maximum values of means (Figure $2 a, b$ ). Cell size was proportional to the granularity. Therefore, because the cell sizes werehigh, the granularity was also large. Microalgae were also routinely evaluated for cellular dry weight (CDW) to highlight biomass productivity. Therefore, determination of cell granularity seems important for understanding biomass production capacity.

The granularity can change in terms of growth phases such as early and late exponential stages. The cells used in this study were mixed at different growth phases to reveal a general pattern of cell morphology. A study revealed the differences in CDW between some microalga [1], but to the best of knowledge, there is no study showing the cell size differences through cell granularity by SSC dot plot of flow cytometry analysis.
Some studies use microscopy to understand the sizes of cells [21]. In this scenario, high-resolution microscopy equipped with at least $63 \mathrm{X}$ objective might be practical for better visualization of microalgae cells. Therefore, we suggest that microscopies with higher performance could be preferred to perform such comparison comprehensively.

The cellular autofluorescence pattern of the cells was also examined by the implementation of different fluorescence channels. As control, the fluorescence amount of both PBS and microalgae media were firstly detected at a similar range with the cells. We identified that, at FL2-H, PBS is a more suitable environment to run cells in terms of a relatively low level of autofluorescence compared to TAP medium (Figure 3a, b). The lowest fluorescence of both microalgae cells was detected at the FL2-H channel; however, the highest was identified at FL3-H (Figure 3c, d). Experiments need to be adjusted by the subtraction of the mean of fluorescence intensity (MFI) of PBS or TAP medium from the MFI of cells. Image-based [22] or standard flow cytometry [16] are in use to analyze cellular components of microalgae. However, the interference of autofluorescence with the detection of the specific markers of interest was overlooked.

The chlorophyll content of microalga is one of the main sources of cellular autofluorescence, and fluorescence is used to be measured in cells detected at forward and side scatter plots [23]. As similar, a typical automated cell counter was further optimized for the facilitated quantification of chlorophyll content in microalgae [24]. Nevertheless, our previous work illustrated that although automated cell counter was not favorably effective, flow cytometry-based analysis was revealed as the best analytical instrument to work with microalgae [25], as also commonly stressed by other researchers [26-28]. 
Flow cytometry is used not only for the detection of fluorescence labeled-components, but also viability detection, biomass estimation, population heterogeneity detection, cell sorting and isolation of axenic algal cultures $[29,30]$. Therefore, the elucidation of

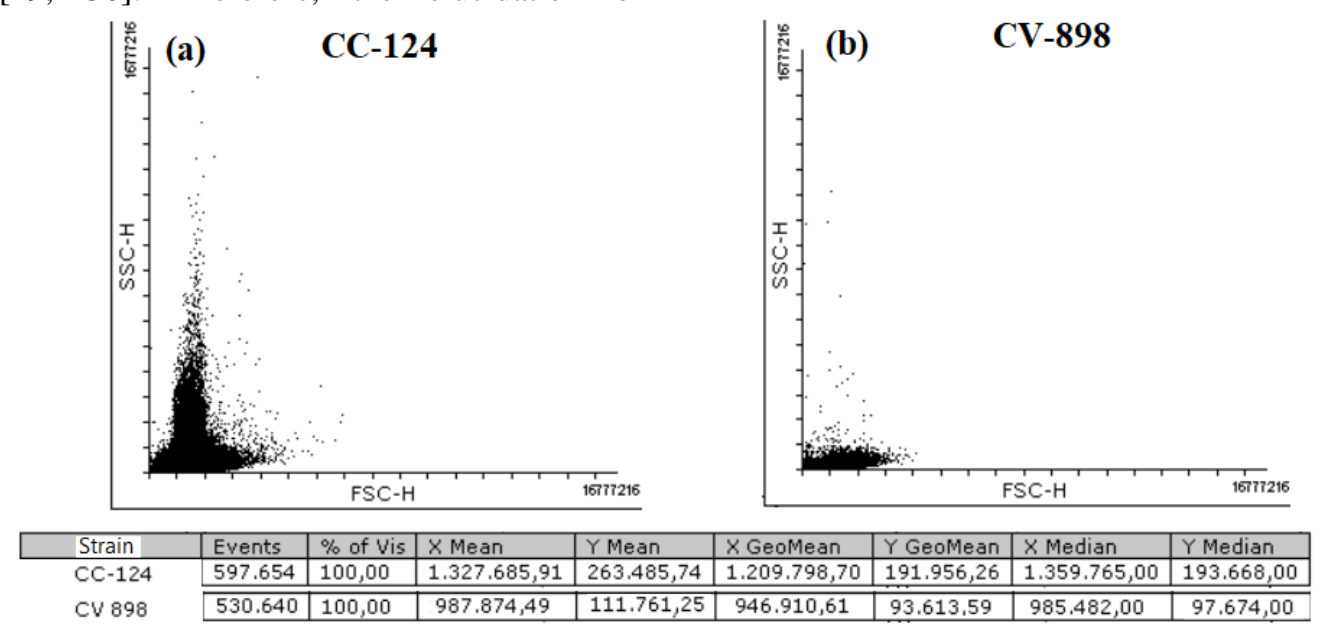

autofluorescence patterning in model microalgae is detected by flow cytometry. Recently, the ontological pattern of autofluorescence was also considered to improve spectroscopic methods [31]. In parallel, crucial

Figure 2. The morphological features of CC-124 (a) and CV-898 (b) by FSC (forward scatter) and SSC (side scatter) representing cell size and granularity, respectively ( $\mathrm{X}$ and $\mathrm{Y}$ axes were adjusted to 16 million unit).

(i)

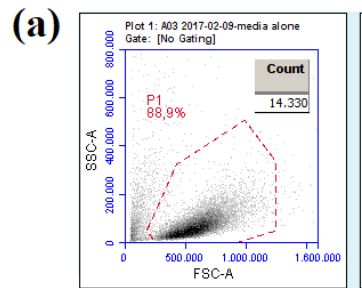

(b)

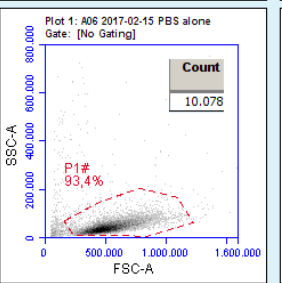

(c)

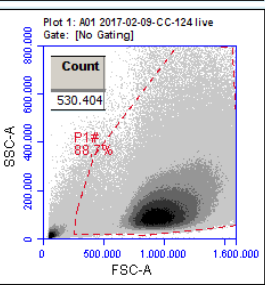

(d)

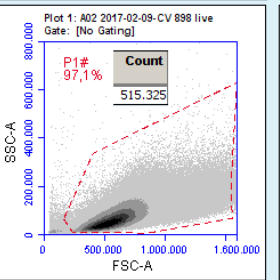

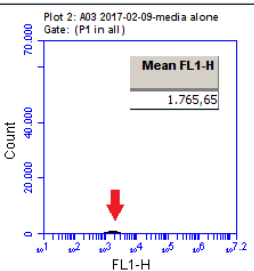
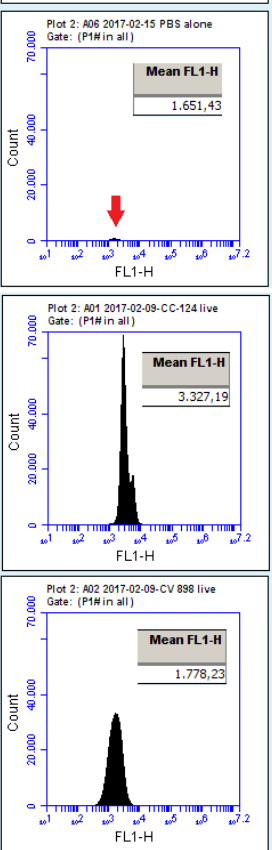

(ii)
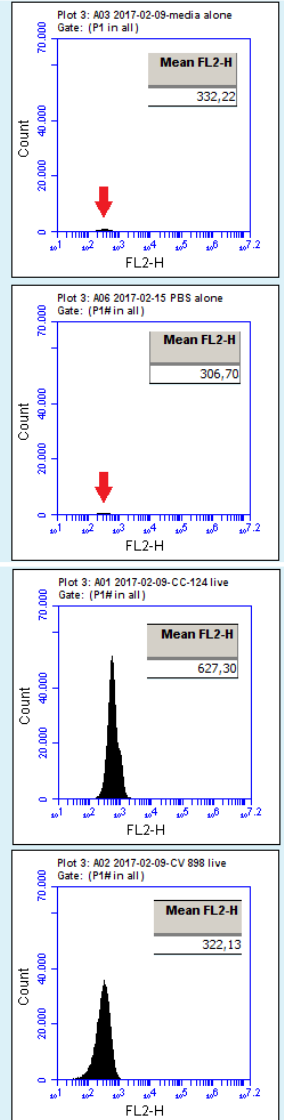

(iii)
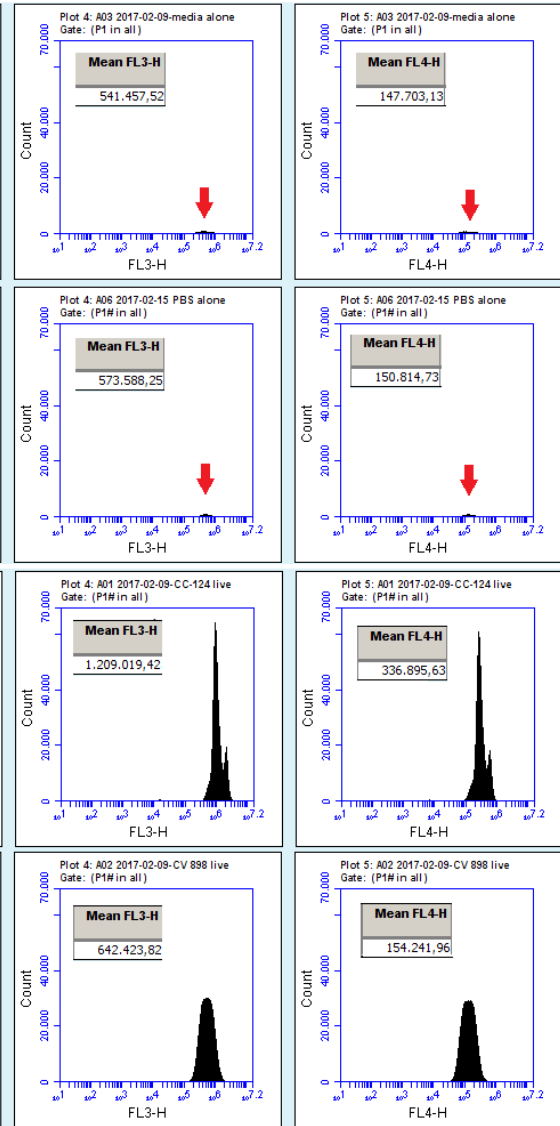

(iv)

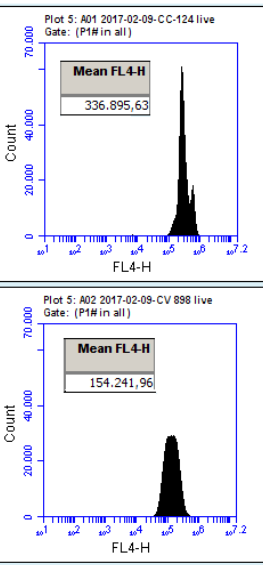

Figure 3. The autofluorescence patterns of TAP medium (a), and PBS buffer (b), CC-124 (c) and CV-898 microalgae cells (d) along with the fluorescence channels $(i-i v)$. (X and Y-axes in cell size (FSC) and granularity (SSC plots) were adjusted to 1.6 million unit). 

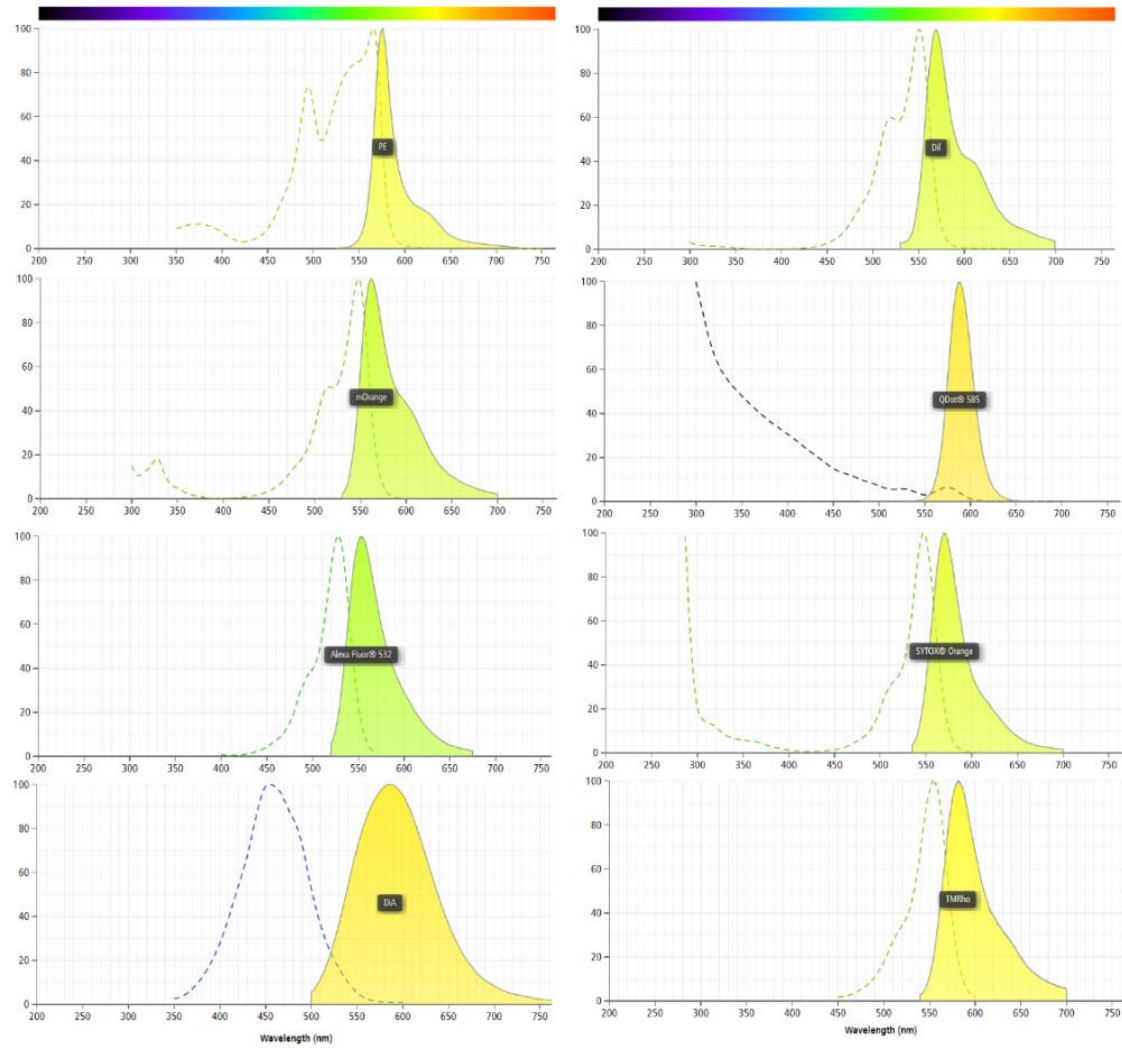

Figure 4. Example wavelengths of some fluorophores given in Table 2.

Table 2. Recommended fluorophores for the signal detection by FL2 channel.

\begin{tabular}{|l|l|}
\hline PE & BVU563 \\
\hline mOrange & SYTOX $®$ Orange \\
\hline Alexa Flour $\AA 532$ & TMRho \\
\hline DiA & Kusabira-Orange \\
\hline Dil & mStrawberry \\
\hline QDot $₫ 565$ & Cy3.5 \\
QDot $₫ 585$ & Cy3B \\
QDot $₫ 605$ & Cy3 \\
QDot $₫ 625$ & \\
\hline
\end{tabular}

in terms of the optimization of labeling methods electrophoresis was shown to be a parameter for the investigation of microalgal diversity [32].

Based on the literature, there is no reported study revealing the autofluorescence distributions of microalgae, in particular for both CC-124 and CV-898. These microalgae are of model organisms in industrial biotechnology towards to production of various valueadded compounds, proteins, vaccines, biofuels and other bioproducts. Industrially crucial lipids and recombinant proteins as microalgae bioproducts are labeled by fluorophores and flow cytometry can provide costeffective and handy outputs to disclose these labels and the total amount of bioproducts.
The current findings also suggest that the best strategy to perform any flow cytometry based measurements on both CC-124 and CV-898 model microalgae is to select a fluorophore emitted at fluorescence channel-2. Table 2 exemplifies some of such representative fluorophores. On the other hand, Figure 4 displays the wavelength distributions of some fluorophores given in Table 2, emitting specifically at FL2-H (Figure 4).

\section{Conclusion}

We found that the minimum fluorescence signal from both model microalgae strains was obtained at only fluorescence channel-2 (FL2-H) of 4 channels. In other words, the obtained results suggest that the most suitable fluorophores for CC-124 and CV-898 cells are the fluorophores emitting at FL2-H. In conclusion, the specific label of interest does not mix with natural signals in both CC-124 and CV-898 cells when the reported results are in consideration. The current knowledge will certainly be practical for the quantitative production of various biomaterials during the versatile biotechnological applications of microalgae.

\section{Acknowledgement}

This work was supported by Scientific Research Projects Coordination Unit of Karadeniz Technical University. Project number: FAY-2016-5755. The author thanks to Ramazan ÇAKMAK due to the preparations of microalgae cell cultures and to Prof. Ersan KALAY 
allowing to utilize of flow cytometry instrument at the Department of Medical Biology at KTU.

\section{Author's Contributions}

Ugur Uzuner: The study was hypothesized, designed, performed and written by Ugur Uzuner.

\section{Ethics}

Authors declare that there are no ethical issues related to publication of this manuscript. The performed study is out of scope of any ethical issues.

\section{References}

1. Abomohra AE-F, Wagner M, El-Sheekh M, Hanelt D (2013) Lipid and total fatty acid productivity in photoautotrophic fresh water microalgae: screening studies towards biodiesel production. J Appl Phycol 25:931-936. https://doi.org/10.1007/s10811-012-9917-y

2. Shuba ES, Kifle D (2018) Microalgae to biofuels: 'Promising' alternative and renewable energy, review. Renewable and Sustainable Energy Reviews 81:743-755. https://doi.org/10.1016/j.rser.2017.08.042

3. Hossain N, Mahlia TMI, Saidur R (2019) Latest development in microalgae-biofuel production with nano-additives. Biotechnology for Biofuels 12:125. https://doi.org/10.1186/s13068-019-1465-0

4. Torres-Tiji Y, Fields FJ, Mayfield SP (2020) Microalgae as a future food source. Biotechnology Advances 41:107536. https://doi.org/10.1016/j.biotechadv.2020.107536

5. Fayyaz M, Chew KW, Show PL, et al (2020) Genetic engineering of microalgae for enhanced biorefinery capabilities. Biotechnology Advances 43:107554. https://doi.org/10.1016/j.biotechadv.2020.107554

6. Kwon YM, Kim KW, Choi T-Y, et al (2018) Manipulation of the microalgal chloroplast by genetic engineering for biotechnologica utilization as a green biofactory. World J Microbiol Biotechnol 34:183. https://doi.org/10.1007/s11274-018-2567-8

7. Yan N, Fan C, Chen Y, Hu Z (2016) The Potential for Microalgae as Bioreactors to Produce Pharmaceuticals. International $\begin{array}{llll}\text { Journal of } & \text { Molecular } & \text { Sciences } & \end{array}$ https://doi.org/10.3390/ijms17060962

8. Jha D, Jain V, Sharma B, et al (2017) Microalgae-based Pharmaceuticals and Nutraceuticals: An Emerging Field with Immense Market Potential. ChemBioEng Reviews 4:257-272. https://doi.org/10.1002/cben.201600023

9. Szpyrka E, Broda D, Oklejewicz B, et al (2020) A Non-Vector Approach to Increase Lipid Levels in the Microalga Planktochlorella nurekis. Molecules 25:. https://doi.org/10.3390/molecules25020270

10. U NM, Mehar JG, Mudliar SN, Shekh AY (2019) Recent Advances in Microalgal Bioactives for Food, Feed, and Healthcare Products: Commercial Potential, Market Space, and Sustainability. Comprehensive Reviews in Food Science and Food Safety 18:18821897. https://doi.org/10.1111/1541-4337.12500

11. Niccolai A, Chini Zittelli G, Rodolfi L, et al (2019) Microalgae of interest as food source: Biochemical composition and digestibility. Algal Research 42:101617. https://doi.org/10.1016/j.algal.2019.101617
12. Sandmann M, Schafberg M, Lippold M, Rohn S (2018) Analysis of population structures of the microalga Acutodesmus obliquus during lipid production using multi-dimensional single-cell analysis. Scientific Reports 8:6242. https://doi.org/10.1038/s41598018-24638-y

13. Yuan D, Zhao Q, Yan S, et al (2019) Sheathless separation of microalgae from bacteria using a simple straight channel based on viscoelastic microfluidics. Lab Chip 19:2811-2821. https://doi.org/10.1039/C9LC00482C

14. Bilican I, Bahadir T, Bilgin K, Guler MT (2020) Alternative screening method for analyzing the water samples through an electrical microfluidics chip with classical microbiological assay comparison of P. aeruginosa. Talanta 219:121293. https://doi.org/10.1016/j.talanta.2020.121293

15. Guler MT, Bilican I (2018) Capacitive detection of single bacterium from drinking water with a detailed investigation of electrical flow cytometry. Sensors and Actuators A: Physical 269:454-463. https://doi.org/10.1016/j.sna.2017.12.008

16. da Silva TL, Reis A, Medeiros R, et al (2009) Oil production towards biofuel from autotrophic microalgae semicontinuous cultivations monitorized by flow cytometry. Appl Biochem Biotechnol 159:568-578. https://doi.org/10.1007/s12010-008-8443-5

17. Benson RC, Meyer RA, Zaruba ME, McKhann GM (1979) Cellular autofluorescence--is it due to flavins? J Histochem Cytochem 27:44-48. https://doi.org/10.1177/27.1.438504

18. Uzuner-Celik S, Peters, L, O’Neill C (2016) Quenching of cellular autofluorescence is necessary for specific detection of DNA methylation by flow cytometry compared to microscopy-based analysis. In: FEBS Journal. FEBSPRESS, Kusadasi, TURKEY, pp 249-250

19. Patel A, Antonopoulou I, Enman J, et al (2019) Lipids detection and quantification in oleaginous microorganisms: an overview of the current state of the art. BMC Chemical Engineering 1:13. https://doi.org/10.1186/s42480-019-0013-9

20. Terashima M, Freeman ES, Jinkerson RE, Jonikas MC (2015) A fluorescence-activated cell sorting-based strategy for rapid isolation of high-lipid Chlamydomonas mutants. Plant J 81:147-159. https://doi.org/10.1111/tpj.12682

21. Nezhad FS, Mansouri H (2019) Induction of Polyploidy by Colchicine on the Green Algae Dunaliella salina. Russian Journal of Marine Biology. https://doi.org/10.1134/S1063074019020093

22. Jeon S-M, Kim JH, Kim T, et al (2015) Morphological, Molecular, and Biochemical Characterization of Monounsaturated Fatty Acids-Rich Chlamydomonas sp. KIOST-1 Isolated from Korea. $J$ Microbiol Biotechnol 25:723-731. https://doi.org/10.4014/jmb.1412.12056

23. Takahashi T (2019) Routine Management of Microalgae Using Autofluorescence from Chlorophyll. Molecules 24:4441. https://doi.org/10.3390/molecules24244441

24. Takahashi T (2018) Applicability of Automated Cell Counter with a Chlorophyll Detector in Routine Management of Microalgae. Scientific Reports 8:4967. https://doi.org/10.1038/s41598-018-23311-

25. Koç E, Çelik-Uzuner S, Uzuner U, Çakmak R (2018) The Detailed Comparison of Cell Death Detected by Annexin V-PI Counterstain Using Fluorescence Microscope, Flow Cytometry and Automated Cell Counter in Mammalian and Microalgae Cells. Journal 
of Fluorescence 28:1393-1404. https://doi.org/10.1007/s10895-0182306-4

26. Sonowal S, Chikkaputtaiah C, Velmurugan N (2019) Role of flow cytometry for the improvement of bioprocessing of oleaginous microorganisms. Journal of Chemical Technology \& Biotechnology 94:1712-1726. https://doi.org/10.1002/jctb.5914

27. Bodénès P, Wang H-Y, Lee T-H, et al (2019) Microfluidic techniques for enhancing biofuel and biorefinery industry based on microalgae. Biotechnology for Biofuels 12:33 https://doi.org/10.1186/s13068-019-1369-z

28. Markina ZhV (2019) Flow Cytometry as a Method to Study Marine Unicellular Algae: Development, Problems, and Prospects. $\begin{array}{llll}\text { Russ } & J & \text { Mar } & \text { Biol }\end{array}$ https://doi.org/10.1134/S1063074019050079

29. Hyka P, Lickova S, Přibyl P, et al (2013) Flow cytometry for the development of biotechnological processes with microalgae. Biotechnology https://doi.org/10.1016/j.biotechadv.2012.04.007

30. Reimann R, Zeng B, Jakopec M, et al (2020) Classification of dead and living microalgae Chlorella vulgaris by bioimage informatics and machine learning. Algal Research 48:101908. https://doi.org/10.1016/j.algal.2020.101908

31. Hadady H, Redelman D, Hiibel SR, Geiger EJ (2016) Continuous-flow sorting of microalgae cells based on lipid content by high frequency dielectrophoresis. AIMS Biophysics 3:398 https://doi.org/10.3934/biophy.2016.3.398

32. Grigoryeva N (2019) Self-Fluorescence of Photosynthetic System: A Powerful Tool for Investigation of Microalgal Biologica Diversity. Microalgae - From Physiology to Application. https://doi.org/10.5772/intechopen.88785 